\title{
THE NEW METHOD OF SURFACE-ASSISTED COF SYNTHESIS FOR OPTICAL SENSOR MODIFICATION
}

\author{
1,2Elena MILIUTINA, ${ }^{1}$ Polina BAINOVA, ${ }^{1}$ Vasilii BURTSEV, ${ }^{1,2}$ Anna ZABELINA, ${ }^{1}$ Vaclav ŠVORČíK, \\ 1,2Oleksiy LYUTAKOV
}

1VŠCHT - University of Chemistry and Technology, Prague, Czech Republic, EU, Iyutakoo@vscht.cz

${ }^{2}$ Tomsk Polytechnic University, Research School of Chemistry and Applied Biomedical Sciences, Tomsk, Russian Federation

https://doi.org/10.37904/nanocon.2020.3712

\begin{abstract}
Covalent organic frameworks (COFs) are an emerging class of crystalline networks that are covalently built from organic components and featured by merits of low density, well-defined pore aperture, large surface area, inherent porosity, facilely tailored functionality, and ordered channel structure. Among other applications, COF compounds can be utilized for $\mathrm{CO}_{2}$ detection. It Is known that the preparation of COFs compounds is commonly associated with hard experimental conditions (high pressure, inert atmosphere, and high temperature). In this work, we suggest an alternative method of COFs under normal pressure and temperature. The experimental concept implements the surface-assisted COF growth. The created surface-immobilized COF structures were subsequently used for measurement of $\mathrm{CO}_{2}$ presence.
\end{abstract}

Keywords: Optical fibers, COFs, plasmons, selectivity

\section{INTRODUCTION}

Covalent organic frameworks (COF) are a new class of crystalline porous organic polymers with controlled porosity and highly ordered structure $[1,2]$. Today, COFs have found a wide range of applications in catalysis, separation and absorption gases [3]. However, the manufacture of COF requires difficult experimental conditions and is quite expensive [4,5]. In addition, the formation of COF layer on functional materil or devices surface still remain a challenging task [6,7]. So, the development of a technology for producing COFs on the solid surface(s) is urgently required. The main goal of this work is to develop the simple and scalable procedure for COF synthesis on directly the sensor surface and subsequent utilization of created hybrid materials for ClOs sensing.

\section{EXPERIMENTAL}

\subsection{Materials}

Gold targets for metal deposition (purity of metals, $4 \mathrm{~N}$ ) were purchased from Deionized water, methanol (reagent grade, $\geq 99.8 \%$ ), acetone (97\%), were purchased from Sigma-Aldrich and used without further purification. 4-cyanobenzenediazonium tosylate (ADT-CN) was prepared according to published procedure [3]. CO2 gas was obtained from SIAD.

\subsection{Samples preparation}

The deposition of Au was accomplished from Au target (purity of $99.99 \%$, provided by Safina, Czech Republic). At the first stage, covalent organic structures of the CTF-1 (carbon triazine framework) type were obtained on the sensor surface (Figure 1). First, the samples were spontaneously modified by soaking in a $3 \mathrm{mM}$ freshly 


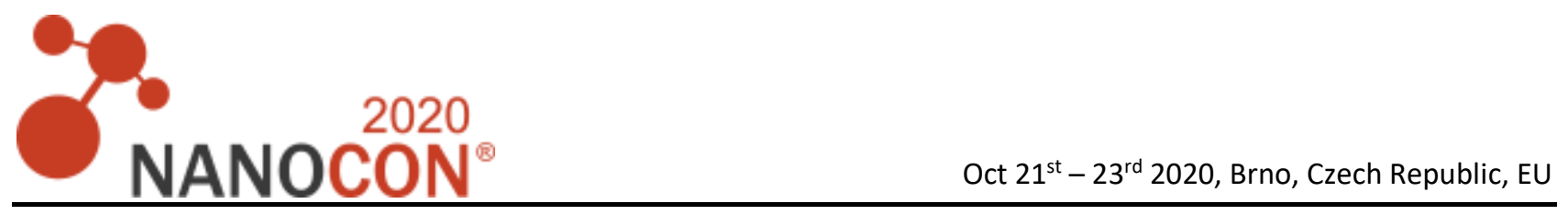

prepared aqueous solution of ADT-CN for $30 \mathrm{~min}$. After modification, the samples were washed with water, methanol, and acetone for 20 minutes and dried in a desiccator for 2 hours. At the next stage, the COF building units were dissolved in chloroform with addition of activation agent and samples were immersed in this solution for $3 \mathrm{~h}$.

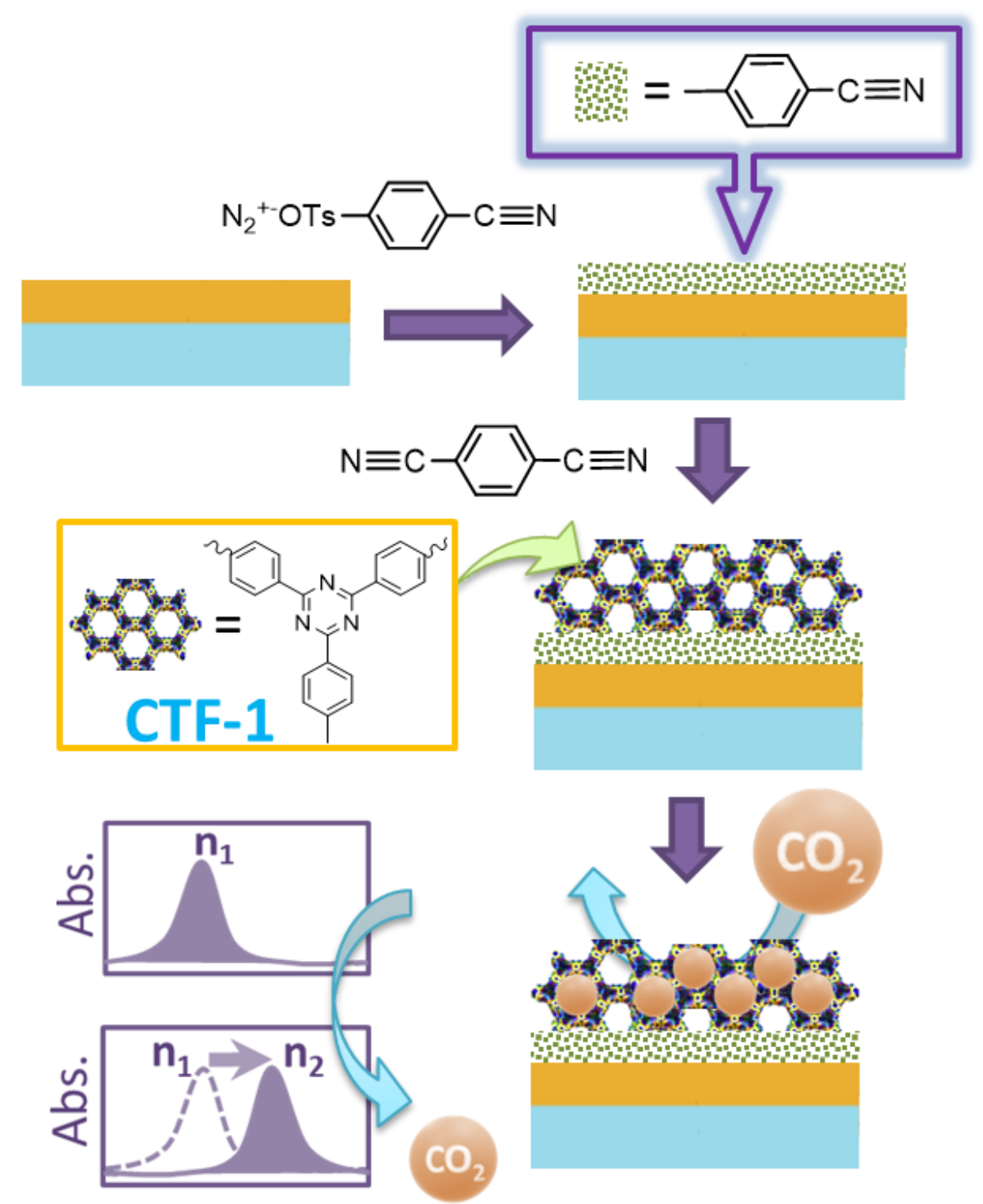

Figure 1 Schematic representation of the fabrication of a sensor with a sensitive surface in the form of CTF-1 covalent organic structures

\subsection{Measurement Techniques}

The X-ray photoelectron spectroscopy (XPS) was performed using an Omicron Nanotechnology ESCAProbeP spectrometer fitted with monochromated AI K Alpha X-ray source working at $1486.6 \mathrm{eV}$.

Raman spectra were measured on ProRaman-L spectrometer (Laser power $35 \mathrm{~mW}$ ) Raman spectrometer with $785 \mathrm{~nm}$ excitation wavelengths. Spectra were measured 30 times, each of them with $3 \mathrm{~s}$ accumulation time.

UV-Vis absorbance spectra of the samples were measured using a HR2000 (Ocean Optics) spectrometer in 400-800 nm wavelengths range using the AvaLight-DHS light source (Avantes). 


\section{RESULTS AND DISCUSSION}

Successful growth of CTF-1 has been verified using XPS, Raman and UV-Vis methods. Figure 2 (parts A, B, C) shows the XPS spectra of pristine surface, surface grafted with - $\mathrm{Ph}-\mathrm{CN}$ and final samples with immobilized CTF-1 layer. The corresponded table (part D) shows the change in the concentration of surface elements depending on the step of surface modification. It is obvious that a gradual increase of concentrations of carbon, oxygen, and nitrogen confirms the shielding of initial sample surface and formation of a CTF-1 layer. We assume that the reaction proceeds according to the following principle:
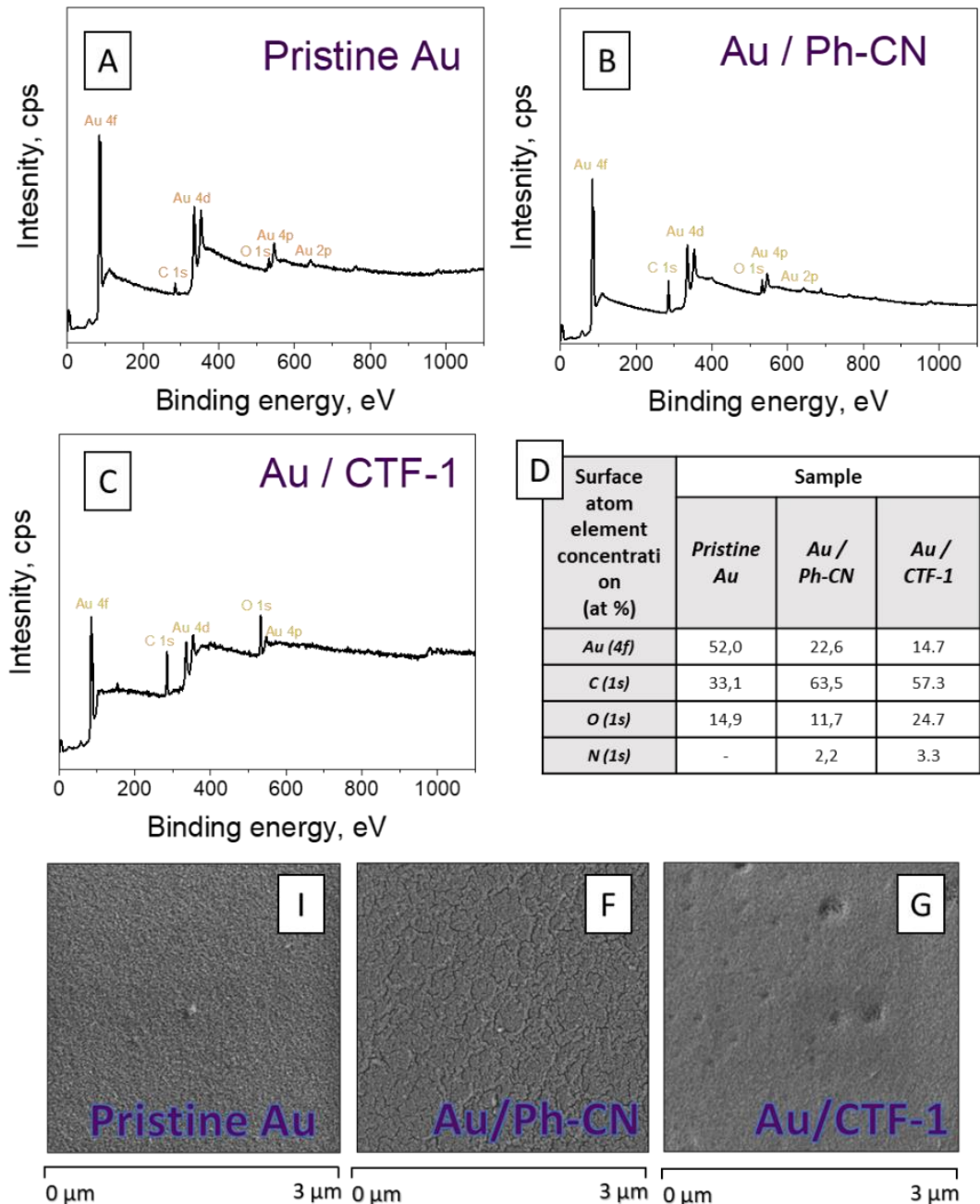

Figure 2 XPS spectra of the surface (A) - pure gold, (B) - gold surface grafted with ADT-CN, (C) gold surface decorated with CTF-1 film. D - Table shows the percentage of elements on the surface. SEM images of the gold surface before $(A)$ and after $(B)$ decoration of the CTF-1 film.

The surface morphology of the samples, before and after decoration with CTF-1 was investigated by SEM. The results are presented in Figure 2 (parts I, F, G) and show that the pristine surface has a slightly rough morphology. This morphology is conserved after the -Ph-CN grafting. After the growth of the CTF-1 film, visible changes in the surface morphology were observed (Figure 2G), indicating that the COF layer is dominantly formed in the surface valleys.

Figure 3 shows the results of Raman spectroscopy (and the corresponding referencing of the spectral positions of the Raman bands). As is evident, the pristine sample surface does not have any spectral features. Grafting of the sample with ADT-CN leads to the appearance of several bands, corresponded to chemical 
fragments of benzene rings and cyano chemical moieties. Further growth of CTF-1 leads to the appearance of several additional Raman bands, the spectral positions of which completely coincide with those in the Raman spectra of CTF-1 powder, also shown in Figure 3B.

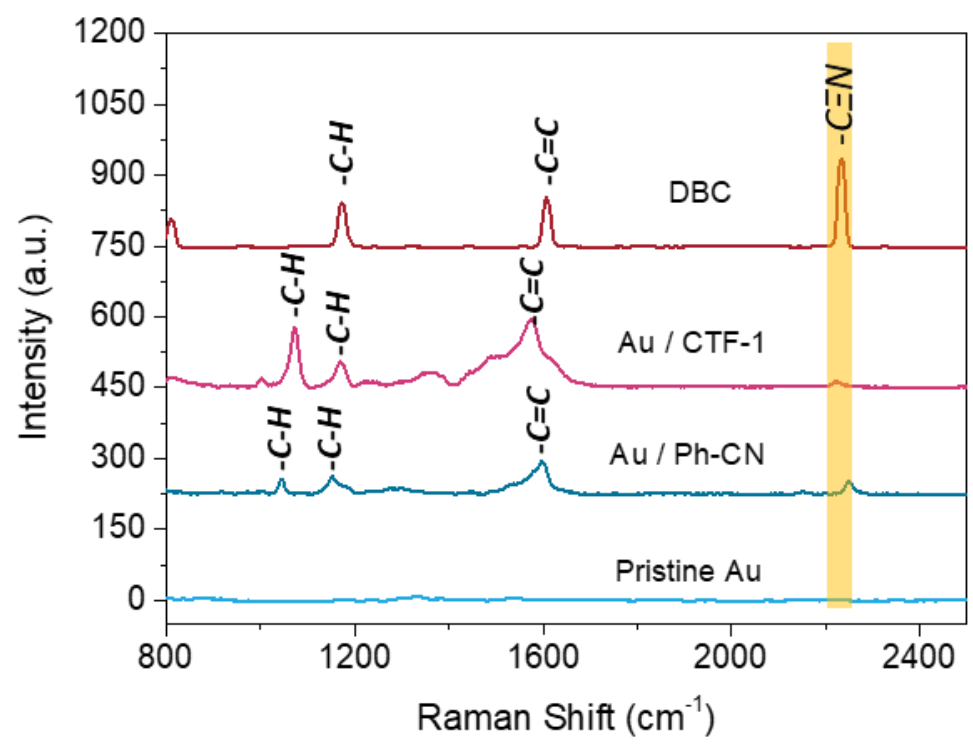

Figure 3 Raman spectra of the surface of primordial gold; a gold surface grafted with ADT-CN and a gold surface decorated with a CTF-1 film

In the next step, sample with CTF-1 were tested for sensitivity to $\mathrm{CO}_{2}$ in water. As seen from Figure 4A, absorption spectrum before and after $\mathrm{CO}_{2}$ purging for 30 minutes did not change the light transmission spectrum. The absorption spectra after COF decoration show a slight broadening of the absorption peak due to $\mathrm{CO}_{2}$ presence. Figure 4B. So, it is also evident that the sensors decorated with COF shows sensitivity to $\mathrm{CO}_{2}$. After blowing with air, the absorption peak returns to its original position, confirming the ability of sensor reutilization.
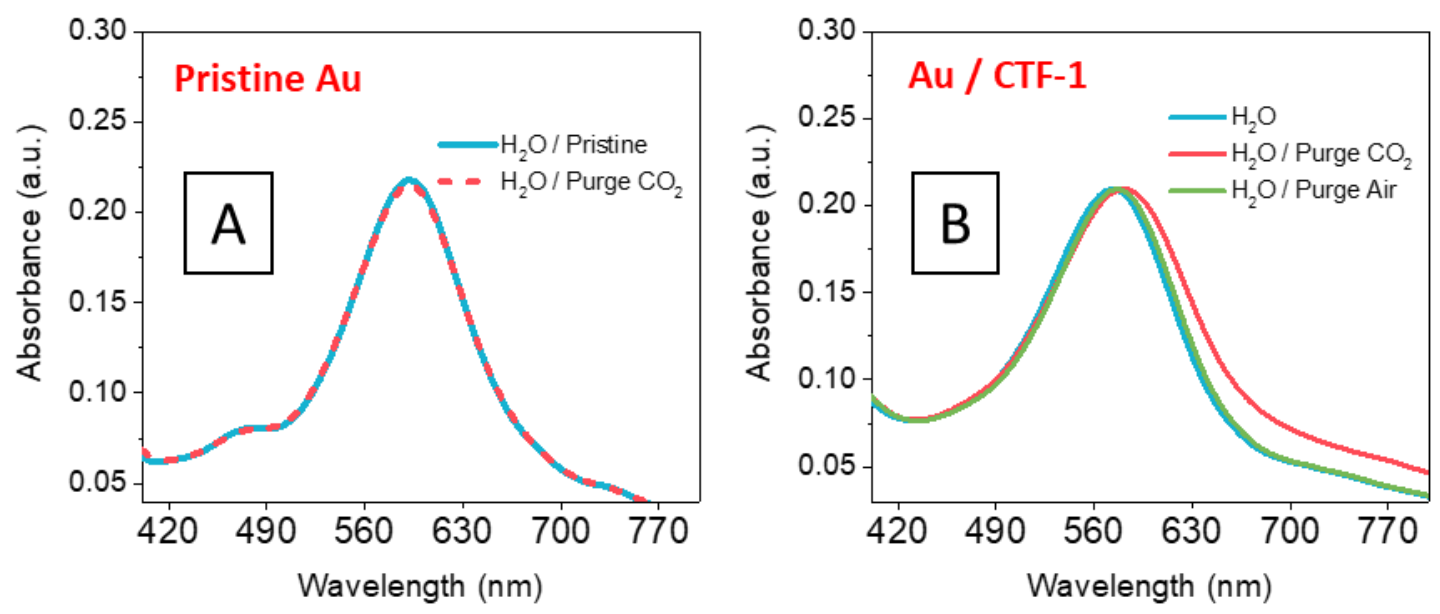

Figure 4 Reaction of an undecorated $(A)$ and decorated COFs $(B)$ sensor to the presence of $\mathrm{CO}_{2}$ in water

\section{CONCLUSION}

In this work, a new method for surface-assisted COF growth is proposed and verified. The COF layer were formed under the ambient condition, without utilization of high temperature or temperature. The COF layer 
were grown on the sensor surface, increasing the affinity of device to $\mathrm{CO}_{2}$. In particular, proposed method allows to identify $\mathrm{CO}_{2}$ presence in water.

\section{ACKNOWLEDGEMENTS}

This work was supported by the project Russian Science Foundation (RSF-19-73-00238) and the project Internal Grant Agency 126882005.

\section{REFERENCES}

[1] GENG, K., HE, T., LIU, R., DALAPATI, S., TAN, K.T., LI, Z., TAO, S., GONG, Y., JIANG, Q., JIANG, D. Covalent Organic Frameworks: Design, Synthesis, and Functions. Chem. Rev. 2020, vol. 120, no. 16, pp. 8814-8933.

[2] YAO, B.-J., ZHANG, X.-M., LI, F., LI, C., DONG, Y.-B. Fe3O4/Porphyrin Covalent Organic Framework Core-Shell Nanospheres as Interfacial Catalysts for Enzymatic Esterification. ACS Appl. Nano Mat. 2020, vol. 3, no. 10, pp. 10360-10368.

[3] GUSELNIKOVA, O., KALACHYOVA, Y., ELASHNIKOV, R., CIESLAR, M., KOLSKA, Z., SAJDL, P., POSTNIKOV, P., SVORCIK, V., LYUTAKOV, O. Taking the power of plasmon-assisted chemistry on copper NPs: Preparation and application of COFs nanostructures for $\mathrm{CO} 2$ sensing in water. Microporous and Mesoporous Mat. 2020, vol. 309, p. 110577.

[4] LI, X., YANG, C., SUN, B., CAI, S., CHEN, Z., LV, Y., ZHANG, J., LIU, Y., Expeditious synthesis of covalent organic frameworks: a review. J. Mater. Chem. A. 2020, vol. 8, no. 32, pp. 16045-16060.

[5] DÍAZ, U., CORMA, A., CORMA, A. Ordered covalent organic frameworks, COFs and PAFs. From preparation to application. Coord. Chem. Rev. 2016, vol. 311, pp. 85-124.

[6] MILIUTINA, E., GUSELNIKOVA, O., SOLDATOVA, N.S., BAINOVA, P., ELASHNIKOV, R., FITL, P., KURTEN, T., YUSUBOV, M.S., ŠVORČ́́K, V., VALIEV, R.R., CHEHIMI, M.M., LYUTAKOV, O., POSTNIKOV, P.S. Can Plasmon Change Reaction Path? Decomposition of Unsymmetrical lodonium Salts as an Organic Probe. J. Phys. Chem. Lett. 2020, vol. 11, no. 14, pp. 5770-5776.

[7] BI, S., YANG, C., ZHANG, W., XU, J., LIU, L., WU, D., WANG, X., HAN, Y., LIANG, Q., ZHANG, F. Twodimensional semiconducting covalent organic frameworks via condensation at arylmethyl carbon atoms. Nat. Commun. 2019, vol. 10, no. 1, p. 2467. 